\title{
A NOVEL METHOD ON FINGERPRINT IMAGE COMPRESSION
}

\author{
Abhilasha A.P ${ }^{\mathbf{1}}$, Laya Tojo ${ }^{2}$ \\ ${ }^{I}$ Student, Electronics and Communication, Oxford College of Engineering, Karnataka, India \\ ${ }^{2}$ Assistant Professor, Electronics and Communication, Oxford College of Engineering, Karnataka, India
}

\begin{abstract}
Here a new technique of fingerprint compression is introduced. Fingerprint compression is a challenging task in storage and communication media because more amount of image has to stored, processed and transformed in many applications. Here the dictionary is obtained from the preprocessed fingerprint patches which are represented as linear combination of sparse method. After the dictionary is formed L-norm minimization is done to find similar blocks and Huffman encoding for effective compression. This proposed method can increase the performance in terms of quality as well as compression. The output is compared with the existing techniques such as JPEG compression method and K-SVD compression method. Finally result analysis is made based on performance parameters such as PSNR and MSE.
\end{abstract}

Keywords: Compression, Huffman Encoding, DCT, PSNR, MSE.

\section{INTRODUCTION}

Consistently huge measure of data is put away, handled and transmitted digitally since the most data are graphical or pictorial in nature the capacity and correspondence necessity are enormous. Strategies for compacting information preceding capacity or transmission are required.

Picture compression addresses the issue of lessening the measure of information required to speak to picture thus the basic standard of diminishment procedure is the evacuation of excess information. For the most part, compression innovations can be classed into lossless and lossy. Lossless compression is a sort of picture compression calculations that permits the first information to be splendidly recreated from the compacted information. Run of the mill picture document organizations of lossless compressionresemble PNG or GIF; it is additionally regularly utilized as a segment inside lossy information pressure innovations. Lossless compression is utilized where it is crucial that the first and the decompressed information be indistinguishable, or where deviations from the first information could be injurious. Lossless compressions techniques might be ordered by kind of information they are intended to pack. Lossy compression innovations more often than not change a picture into another space, quantize and encode its coefficients. Lossy compression is the kind of picture encoding techniques that utilizations vague approximations to speak to the substance. These techniques are utilized to decrease information size for capacity, taking care of, and transmit content. The measure of information diminishment achievable utilizing lossy compression is frequently much higher than through lossless strategies. In lossy change codes, tests of picture are taken, slashed into little sections, changed into another premise space, and quantized. The subsequent quantized qualities are then encoded utilizing entropy coding. There are change based picture compressions strategies have been verifiably inquired about and a few standards have showed up. Two most basic alternatives of change are the Discrete Cosine Transform (DCT) and the Discrete Wavelet Transform (DWT)

Finger impression acknowledgment is exceptionally famous for individual distinguishing proof because of the uniqueness, all inclusiveness, collectability and invariance. Vast volumes of information devour the measure of memory.

Every one of the Technologies utilized as a part of our days present to us a way to confine the entrance to a framework, permitting the passageway just to those persons who know a particular code, claim a card or have decided physic marks. The more mind boggling is the framework, the most troublesome is to be assaulted, despite the fact that it will be more costly and will require more programming and equipment assets. At the point when another validation framework is embedded, it is key a judgment between straightforwardness, cost and productivity, and additionally social adequacy.

In Sparse representation there are basically three steps.They are dictionary construction, fingerprint image compression and encoding. Sparse representation is an atomic representation. Here each image is formed into a column and each column is called as atoms. Sparse representation are more widely applied for special images such as face recognition, fingerprint images etc.Since general images have large amount of data or information in pictorial or graphical form to be compressed. Such images tend to have very large dictionary and hence cannot be sparsely depicited.

\subsection{Dictionary Construction}

First, we construct a training set prior to dictionary construction. Select the fingerprint image, resize the image into $300 * 300$ because if the resolution is larger the computer allotted time also increases and the dictionary becomes extra 
large and hence cannot be sparsely depicted. Hence this size is ideal for fingerprint images. Next the image is divided into a number of patches. Constructing the training set, first a single patch will be added to the empty dictionary. The next patch will be compared and added, if they are similar then we chose the next patch, if no then the patch will be added to the set. This will be continued till the last patch is test for the entire fingerprint image.
Before the sparse dictionary is formed the mean value is calculated and subtract from each pixel. This method of forming dictionary is done by a clustering method called as K-SVD. Then updating the dictionary is done by solving singular value decomposition (SVD).

\subsection{Fingerprint Image Compression}

15

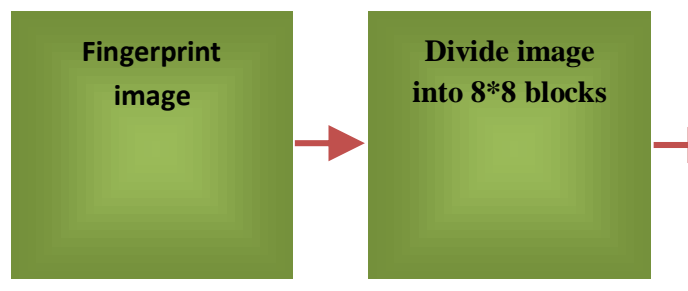

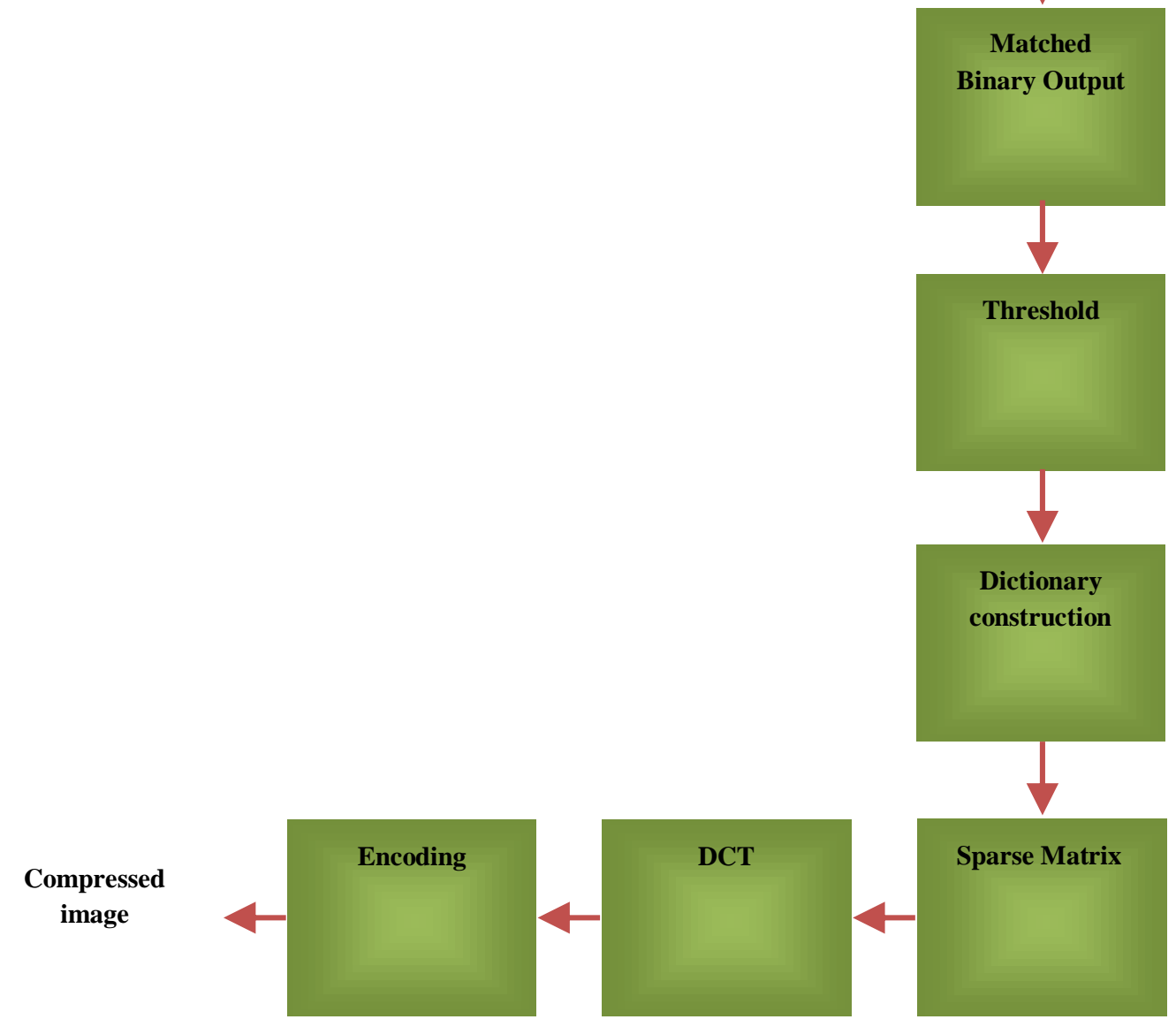

FIG-1: Block Diagram of Compressed Fingerprint Image

The fingerprint image is divided into a number of patches, each patches have like size. However the size has straight approach over the performance of the special fingerprint image. The dictionary should be large enough so as to increase the compression efficiency. In adding up for dividing the image into blocks, the mean of each patch is calculated and subtracted from every other patches. Then 1norm minimization is performed by identifying similar blocks and thresholding is done for those patches whose absolute value is less than the threshold will be retained as zero. Thus we store the mean value, index value and sparsely represented data.

\subsection{Huffman Encoding}

Huffman coding was early devised by a scientist David A. Huffman in 1952. Huffman coding has many unique properties such as follows Huffman coding is an uneven length coding that is each symbols in the code will tend to have a codeword of different length. 
a. They have an exclusive prefix property, where the codeword's preserve their optimality by having different prefix for each symbols. Here no two diverse symbols will have the same prefixed code.

b. Huffman coding is displayed in a binary tree format which helps to understand the coding part of each variables or value in the tree. Finally it reduces the redundant codes and thus the data obtained is the compressed binary output.

\section{ALGORITHM}

1. Reading Fingerprint Image.

2. Converting into blocks.

3. Subtracting Mean with All elements of blocks.

4. Creating an Empty Dictionary. Select the first block as reference block and other blocks as test blocks

5. Match all the blocks using SVD and Norm Minimization

6. Store Mean value, Matched block, and index into dictionary. Repeat as step 5 till the last block.

7. Perform DCT based compression for Matched blocks in the dictionary.

8. Perform Huffman coding and Store Compressed value.

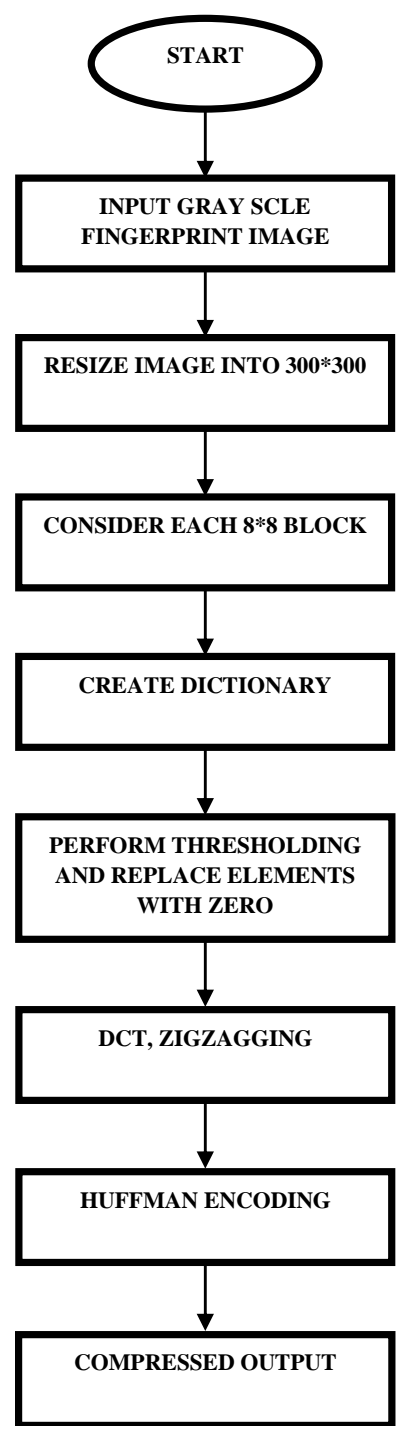

Fig 2. Flowchart

\section{RESULT}

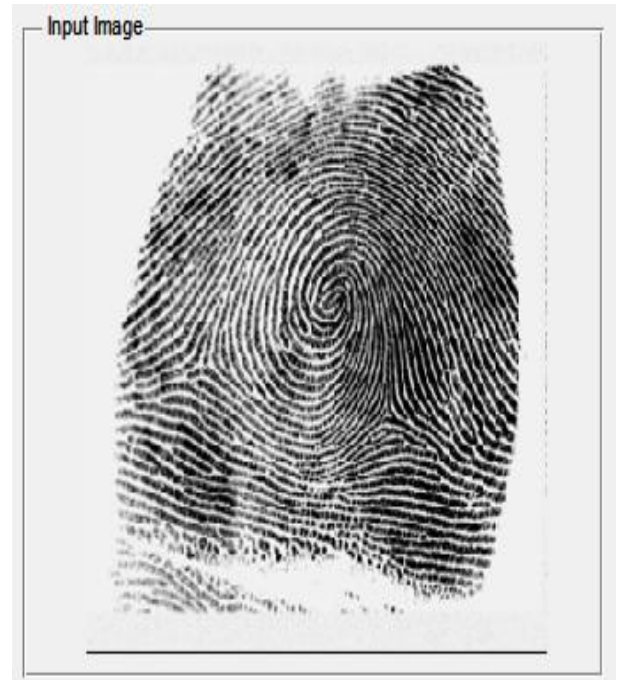

FIG-3: Fingerprint Image Input

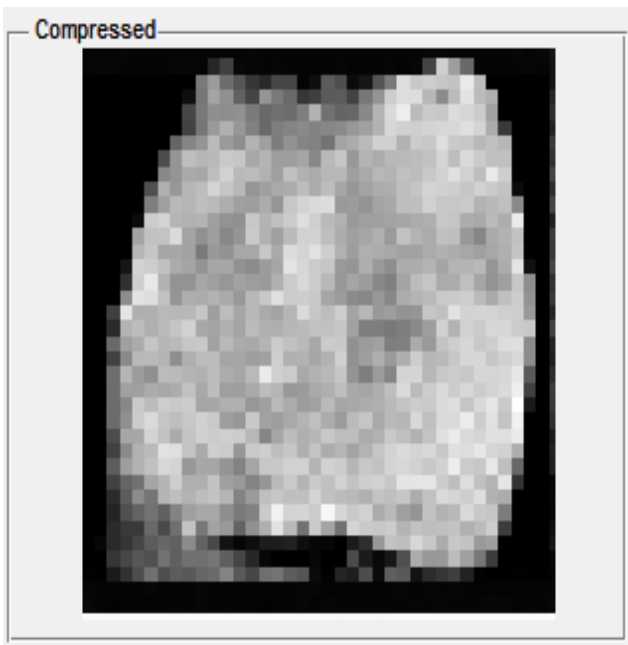

FIG-4: Huffman Compressed Image Output

The above figure shows the compressed size of the fingerprint image using a DCT and Huffman Encoding(proposed method). The compressed image is decompressed to reconstruct the original fingerprint image and PSNR, MSE values are displayed below.

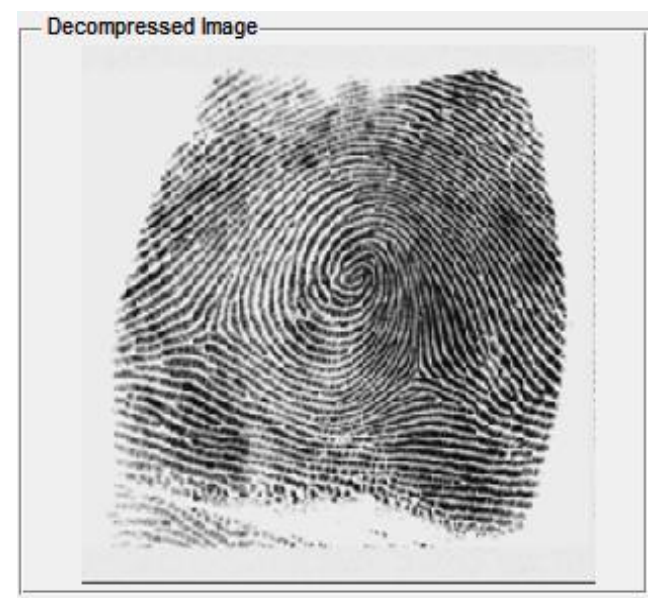

FIG-5: Huffman Decompressed Image Output 
Table-1: PSNR, MSE and Compressed size comparison

\begin{tabular}{|l|l|l|l|}
\hline & $\begin{array}{l}\text { Compressed } \\
\text { size(Kbytes) }\end{array}$ & PSNR & MSE \\
\hline $\begin{array}{l}\text { PROPOSED } \\
\text { METHOD }\end{array}$ & 42.954 & 27.1891 & 124.2142 \\
\hline K-SVD & 58.6931 & 22.4081 & 373.4794 \\
\hline JPEG & 90.755 & 2.9171 & 33217.877 \\
\hline
\end{tabular}

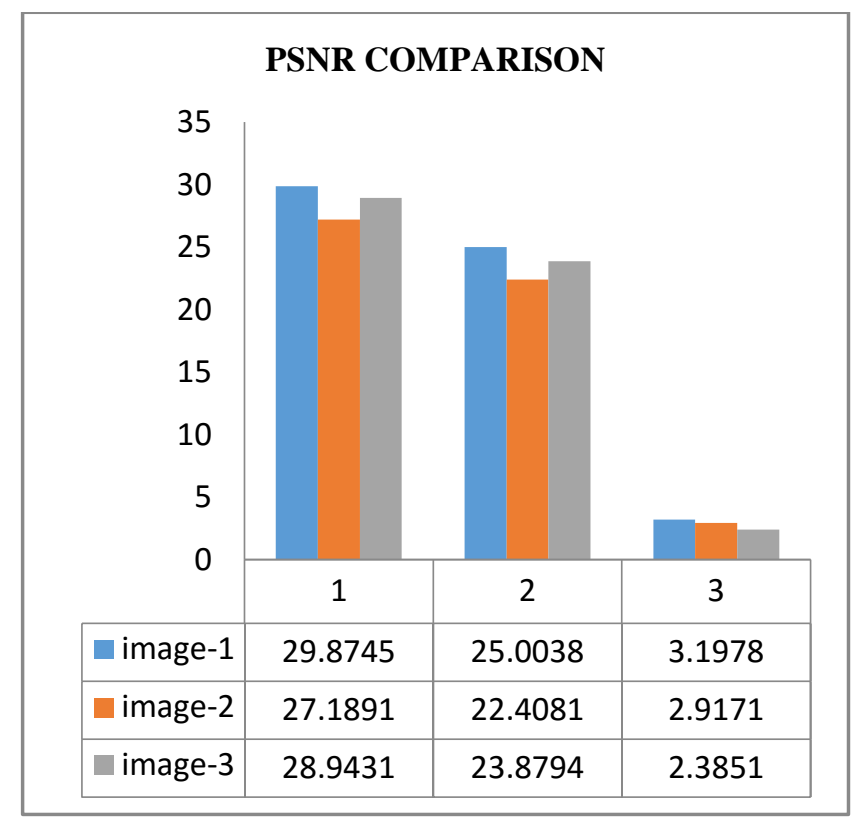

FIG-6: PSNR Comparison

The graphs are plotted based on the differencesin the PSNR,MSE and Compressed size of three different set of fingerprint images.Hence the graph plotted shows that the proposed model has a better performance when compared to existing model and JPEG compression method.

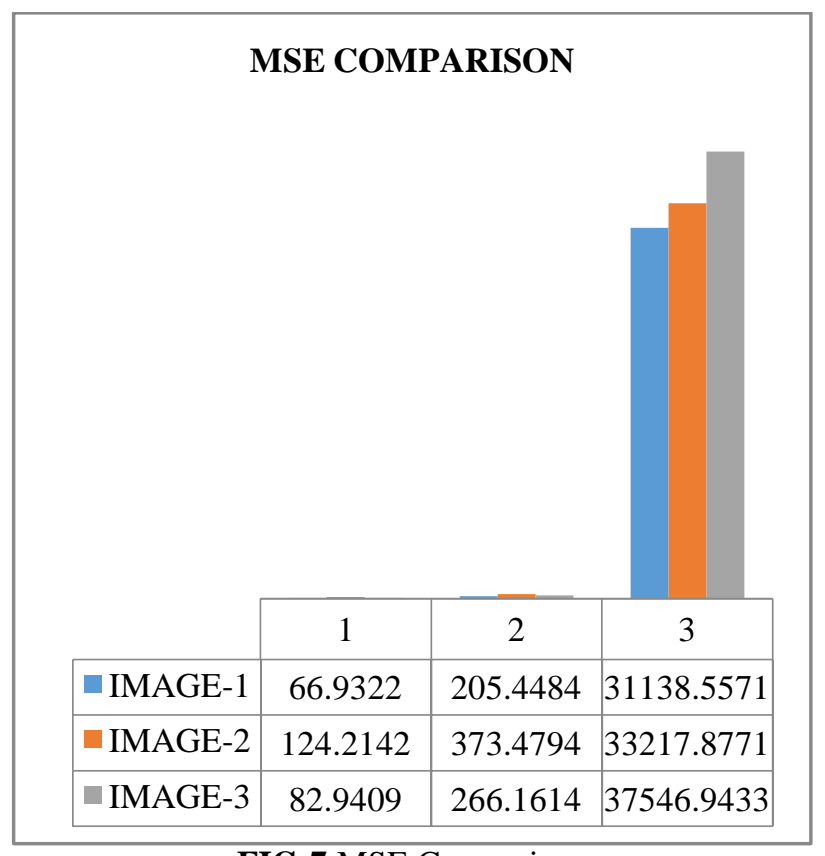

FIG-7:MSE Comparison

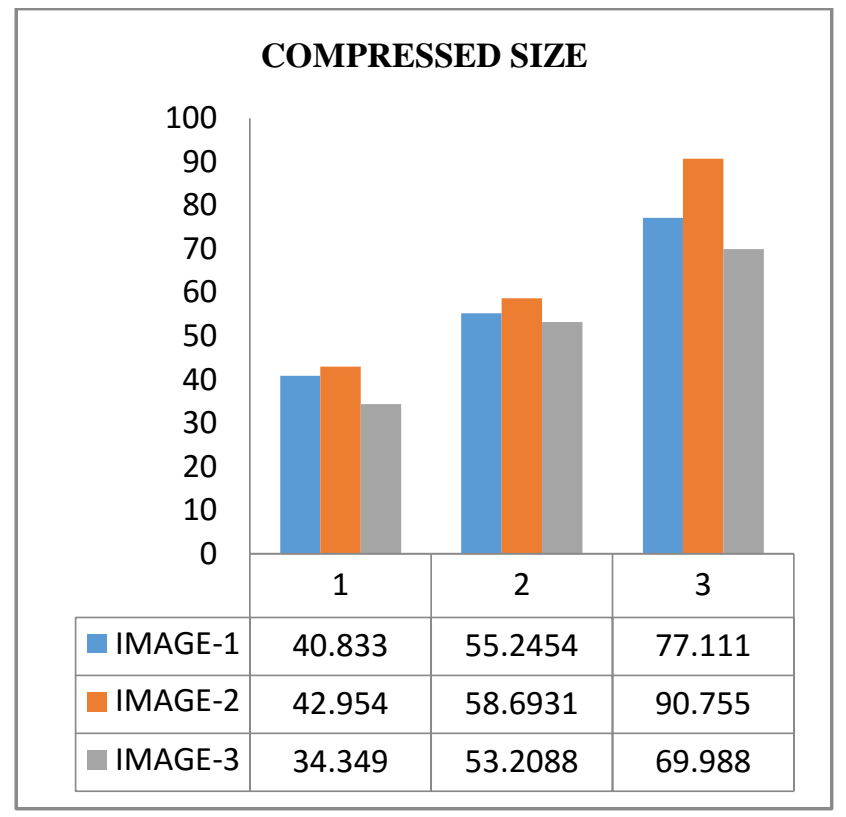

FIG-8:Compressed size comparison

\section{CONCLUSION}

This paper introduced a novel direct fingerprint image compression method based on sparse representation the different compression techniques adapted to compress the fingerprint images are reviewed and their Performance are compared

On the other side, our result analysis shows the comparison in term which is of PSNR and MSE obtained using mathematical method and MATLAB. PSNR and MSE comparison are done based on three sets of database consisting of few samples of fingerprint images. So we conclude that the fingerprint images in terms of PSNR, compressed size and MSE show that the fingerprint images are well compressed using our proposed method in comparison to K-SVD compression method and JPEG standard compression method.

\section{REFERENCES}

[1]. S. G. Mallat and Z. Zhang, "Matching pursuits with time frequency dictionaries," IEEE Trans. Signal Process.vol. 41, no. 12, pp. 3397-3415, Dec. 1993.

[2]. S. S. Chen, D. Donoho, and M. Saunders, "Atomic decomposition by basis pursuit," SIAM Rev., vol. 43, no. 1, pp. 129-159, 2001.

[3]. J. Wright, A. Y. Yang, A. Ganesh, S. S. Sastry, and Y. $\mathrm{Ma}$, "Robust face recognition via sparse representation," IEEE Trans. Pattern Anal. Mach. Intell., vol. 31, no. 2, pp. 210-227, Feb. 2009.

[4]. M. Elad and M. Aharon, "Image denoising via sparse and redundant representation over learned dictionaries," IEEE Trans. Image Process., vol. 15, no. 12, pp. 3736-3745, Dec. 2006.

[5]. S. Agarwal and D. Roth, "Learning a sparse representation for object detection," in Proc. Eur. Conf. Compute. Vis., 2002, pp. 113-127. 
[6]. J. Yang, J. Wright, T. Huang, and Y. Ma, "Image super-resolution as sparse representation of raw image patches," in Proc. IEEE Conf. Compute. Vis. Pattern Recognition., Jun. 2008, pp. 1-8.

[7]. K. Skretting and K. Engan, "Image compression using learned dictionaries by RLS-DLA and compared with K-SVD," in Proc. IEEE ICASSP, May 2011, pp. 15171520 .

[8]. O. Bryt and M. Elad, "Compression of facial images using the K-SVD algorithm," J. Vis. Commun. Image Represent.vol. 19, no. 4, pp. 270-282, 2008.

[9]. J. Ferreira and M. A. T. Figueiredo, "Class-adapted image compression using independent component analysis," in Proc. Int. Conf. Image Process., vol. 1. 2003, pp. 625-628.

[10].A.J. Ferreira and M.A.T. Figueiredo, "On the use of independent component analysis for image compression," Signal Process, Image Commun., vol. 21, no. 5, pp. 378-389, and 2006.

[11].P. Paatero and U.Tapper,"Positive matrix factorization: A nonnegative factor model with optimal utilization of error estimates of data values,"Environmetrics, vol. 5, no. 1, pp. 111-126, 1994.

[12].Guangqi, Yonping, Wu, Yang A, Xiao Liu and TiandeGuo,"Fingerprint compression based on sparse representation", vol.23 no.2 Feb 2014.

\section{BIOGRAPHIES}

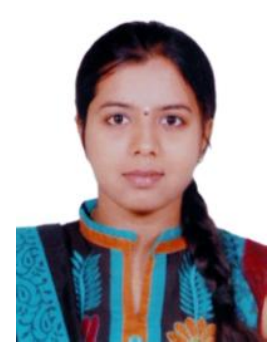

Abhilasha A.P completed her B.E in Electronic \& Communication discipline from Visveswaraya Technological University at East point college of Engineering \& Technology, Bangalore. Currently pursuing Mtech in Digital Electronics \& Communication at Oxford College of Engineering, Bangalore.

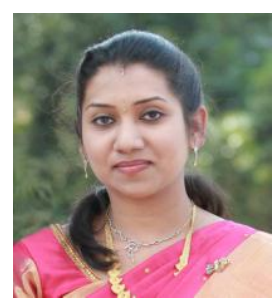

LayaTojo, received her B.Tech degree in Electronics and Communication Engineering from Calicut University and M.Tech Degree in Digital Electronics and Communication Engineering from Visveswaraya Technological University, Belgaum. She is currently working as Assistant Professor in the Department of Electronics and Communication Engineering at The Oxford College of Engineering ,Banagalore. She is currently pursuing her Ph.D degree in image processing. 